EPJ Web of Conferences 19, 08004 (2012)

DOI: $10.1051 /$ epjconf/20121908004

(C) Owned by the authors, published by EDP Sciences, 2012

\title{
The infrared Galactic disk: What have we learned?
}

\author{
E. Churchwell ${ }^{\mathrm{a}}$ \\ University of Wisconsin-Madison
}

\begin{abstract}
A brief overview is given of some of the new insights we have achieved from the Spitzer/GLIMPSE/MIPSGAL surveys of the Galactic plane. Particular emphasis is given to infrared bubbles, rapidly accreting protostars, infrared dark clouds, and diffuse PAH emission. Large scale galactic structure based on the distribution of red clump giants will be discussed by Robert Benjamin in this volume.
\end{abstract}

\section{INTRODUCTION}

Several large-scale near- and mid-infrared surveys of the Milky Way are providing deeper insights into the structure, contents, and physics of the of the Galactic disk; resulting in an evolving new view of our Galaxy. Infrared surveys provide low to zero extinction images of the disk, samples the cool components in the disk, and trace thermal and non-thermal infrared emission mechanisms in the Milky Way. My goal here is to report some of the main new insights we have gleaned from the GLIMPSE/MIPSGAL Spitzer mid-infrared surveys of the Galactic plane.

Hall marks of the IR Galactic plane are: the distribution of the cool, old stellar population; bubbles/HII regions, AGB shells, PNes, \& SNRs; the youngest stages of rapidly accreting massive young stellar objects (YSOs); infrared dark clouds (IRDCs); and diffuse polycyclic aromatic hydrocarbons (PAHs) and thermal dust emission. Robert Benjamin will be discussing large scale galactic structure based on the distribution of red giants (especially red clump giants) from the GLIMPSE/MIPSGAL/ 2MASS surveys. Space and time only permit me to discuss in any detail IR bubbles and rapidly accreting protostars (YSOs). I will very briefly mention some results for IRDCs and diffuse PAHs.

\section{IR BUBBLES}

IR bubbles are among the most spectacular objects in the GLIMPSE/MIPSGAL mosaiked images. They are numerous, over 600 have been identified, and widely distributed in the disk. In twodimensional projections, three dimensional bubbles appear ring-shaped with $8 \mu \mathrm{m}$ (PAH dominated) shells surrounding $24 \mu \mathrm{m}$ thermal dust emitting interiors. Comparison of the IR images with radio continuum surveys, shows very clearly that the radio free-free emission and $24 \mu \mathrm{m}$ thermal dust emission distributions are essentially identical, indicating unequivocally that dust is well mixed with the ionized gas of the HII region.

In high resolution IR and radio continuum images, one can identify two types of HII regions; those that are ionized by stars with strong stellar winds and those that are not. The distinguishing characteristic is the presence or absence of a small central wind-evacuated region around the central star(s), which is apparent by a dip in both $24 \mu \mathrm{m}$ thermal dust emission and radio continuum emission toward the ones with strong winds but not toward those with weak winds.

\footnotetext{
ae-mail: churchwell@astro.wisc.edu
}

This is an Open Access article distributed under the terms of the Creative Commons Attribution-Noncommercial License 3.0, which permits unrestricted use, distribution, and reproduction in any noncommercial medium, provided the original work is properly cited. 


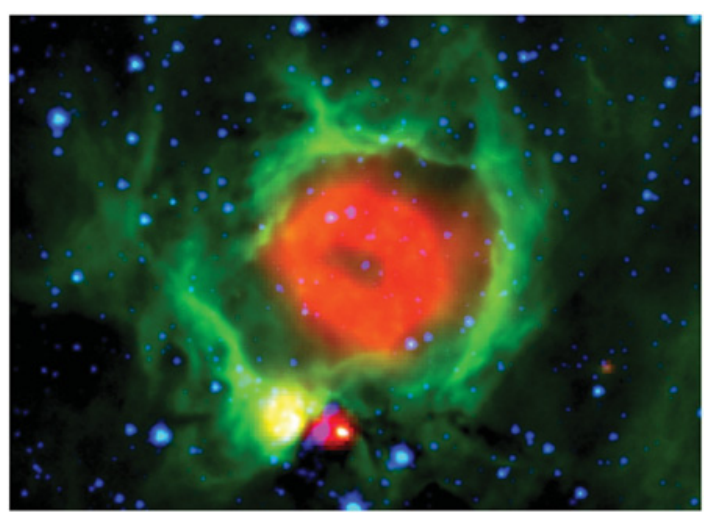

Figure 1. A three-color image of the wind-blown bubble N49. Red is $24 \mu \mathrm{m}$ thermal dust emission. Green is $8 \mu \mathrm{m}$ emission dominated by PAHs, which traces the PDR region of the HII region. Blue is $4.5 \mu \mathrm{m}$, which primarily shows the locations of stars. The central "hole" or depression is due to wind evacuation of dust and gas. Three young stellar objects are located at the bottom edge of the PDR region.

In Figure 1 an image is shown of the prototypical wind-blown bubble N49 [1]. In this figure, the expected central dip (hole) is apparent as well as the PAH (photodissociation region, PDR) shell at $8 \mu \mathrm{m}$. The ratios of Spitzer/IRAC bands that contain PAHs with the $4.5 \mu \mathrm{m}$ band that contains no PAH features clearly show that PAHs are absent within the ionized hydrogen volume. This clearly shows that PAHs are effectively destroyed by photons of energy greater than $13.6 \mathrm{eV}$, but excited by and not destroyed by photons of energy less than $13.6 \mathrm{eV}$ (i.e. those that can pass through the hydrogen ionization front).

In an effort to determine the properties of dust in the HII region and its impact on the evolution of HII regions, [6] used Cloudy [7, 8] to estimate the expected lifetime of dust in a wind-blown HII region and its impact on the temperature of the wind-shocked regions of HII regions. They found that dust temperatures are less than $100 \mathrm{~K}$ over all grain sizes larger than $0.05 \mu \mathrm{m}$ and distances greater than $1 \mathrm{pc}$ from the central star, indicating that dust destruction by thermal evaporation is ineffective. Dust destruction by sputtering was shown to survive for less than $10^{5} \mathrm{yr}$ for grains with radii less than $0.01 \mu \mathrm{m}$, but larger grains can survive for $10^{5}$ to a few times $10^{6} \mathrm{yrs}$ for the largest grains, commensurate with the age of the HII region. It was found that the most important mechanism of removing dust from the HII region was the combined effects of wind drag and radiation pressure, which removes all dust on time scales less than $10^{4} \mathrm{yrs}$ no matter where the dust is released in the nebula. Thus, in a nebula of age $10^{6} \mathrm{yr}$, we should expect to find no dust in the HII region, in contradiction to the observational evidence provided by the $24 \mu \mathrm{m}$ emission distribution. Either the nebula is younger than $10^{4} \mathrm{yrs}$, which would require an expansion rate much greater than predicted by any reasonable model, or a continuous supply of dust is required. [6] suggested that continuous supply of dust could be provided by small, dense cloudlets of ISM that were over-run by the ionization front which slowly release dust grains as the cloudlets photoevaporate. Another source of grains might be the slow release of dust from disks around less massive stars that were formed with the most massive star(s) responsible for ionization of the HII region; this would be like the proplids found near the Trapezium in the Orion nebula. Whatever their origin, it is clear that dust resides in the ionized regions of HII regions based on the close correspondence of $24 \mu \mathrm{m}$ and radio continuum emission.

So what are the consequences of dust mixed within HII regions? First, in the low densities $(\leq 10$ $\mathrm{cm}^{-3}$ ) and high radiation fields expected in the wind- shocked regions of HII regions, dust is expected to be highly positively charged and the dominant coolant of the gas. One of the puzzles of such regions has been why they are not strong X-ray sources. It now seems likely that a relatively small amount of 

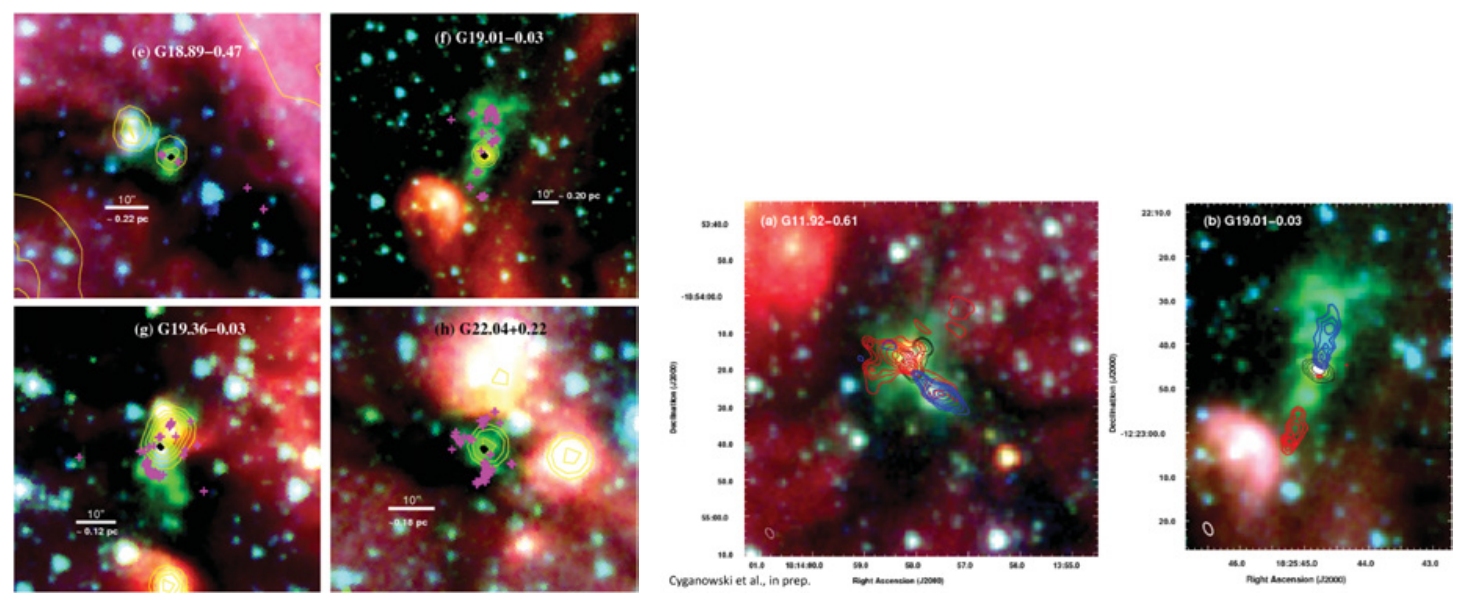

Figure 2. (left): A montage of four EGOs from GLIMPSE images (red-8 $\mu \mathrm{m}$ : green-4.5 $\mu \mathrm{m}$ : blue-3.6 $\mu \mathrm{m}$ ) showing the distribution of thermal $24 \mu \mathrm{m}$ emission (yellow contours), $\mathrm{CH}_{3} \mathrm{OH} 6.7 \mathrm{GHz}$ maser emission (black diamonds), and $44 \mathrm{GHz} \mathrm{CH} \mathrm{CH}_{3} \mathrm{OH}$ masers (magenta crosses). Note that the $24 \mu \mathrm{m}$ and $6.7 \mathrm{GHz}$ masers are centered on the same spot (presumably the central YSO) and the $44 \mathrm{GHz}$ masers are distributed along the extended $4.5 \mu \mathrm{m}$ emission. (center and right): Two examples of EGOs from GLIMPSE images (color code same as Figure 3) with CO bipolar outflows shown in red and blue contours. Note that the CO outflows are oriented along the extended $4.5 \mu \mathrm{m}$ emission.

dust can reduce the gas temperatures from $10^{7-8} \mathrm{~K}$ to $10^{5} \mathrm{~K}$ or less, rendering X-ray emission below the detection limit of Chandra. Thus dust cooling provides a reasonable solution to a long-standing problem. Second, the presence of dust reduces the radius of the HII region so its age is no longer a simple function of radius and initial ambient density. Third, dust cooling reduces the total energy of the nebula, which will significantly affect its evolution. And fourth, the ionization structure of the nebula is likely to look like a nebula ionized by a cooler star than the actual ionizing star due to cooling of the gas and absorption by dust of ionizing photons. The lesson here is that dust plays an important role in the structure and evolution of HII regions and cannot be neglected in realistic models of HII region evolution.

\section{RAPIDLY ACCRETING PROTOSTARS}

The star formation community has long sought a quick and easy way to identify stars in their earliest stages of formation. Generally that has been done by painstakingly studying known regions of star formation or radio maser sources with high spatial resolution and sensitivity to look for evidence of infall, bipolar outflows, or circumstellar disks, all of which require large investments of telescope time and human effort. The GLIMPSE surveys have serendipitously discovered a population of slightly extended $4.5 \mu \mathrm{m}$ excess sources, which have subsequently been shown to be massive protostars in the early stage of rapid accretion well before they have produced a detectable HC or UC HII region. GLIMPSE three-band images including the $4.5 \mu \mathrm{m}$ band labeled as green shows hundreds of slightly extended green objects (EGOs). Figure 2(left) shows a montage of several selected EGOs from the GLIMPSE survey. Further study of EGOs has shown that their MIR spectral energy distributions have a small excess in the $4.5 \mu \mathrm{m}$ band, reside in MIR color-color regions populated by YSOs, are located in IRDCs, are coincident with $\mathrm{CO}$ peaks, have broad $\mathrm{HCO}+$ line wings consistent with infall or outflows, have no detectable free-free emission, and are tightly correlated with $\mathrm{CH} 3 \mathrm{OH}$ masers (both 6.7 and 44 $\mathrm{GHz}$ masers). These are all hallmarks of the youngest stages of protostars. 
Let us now examine the evidence that EGOs are what they are claimed to be, namely massive protostars in the process of rapidly accreting with accompanying strong bipolar outflows. The first question we might ask is: Why do EGOs have excess $4.5 \mu \mathrm{m}$ emission? There is now good spectroscopic evidence that the excess emission is due to shock-excited molecular line emission (due primarily to $\mathrm{H} 2$ and possibly also to some $\mathrm{CO}$ band head emission: [3, 4, 9-11]) produced where bipolar outflows crash into the ambient interstellar medium. Cyganowski [2] obtained high spatial resolution images of $\mathrm{CH}_{3} \mathrm{OH} 6.7$ and $44 \mathrm{GHz}$ masers. The class II $6.7 \mathrm{GHz}$ transition is excited by infrared radiation and the class I $44 \mathrm{GHz}$ transition is collisionally excited. Because of the different excitation mechanisms, these transitions are expected to have different spatial distributions. Indeed, Cyganowski and her collaborators found quite different distributions for the two transitions of the same molecule. In Figure 2 (left) a montage of four EGOs, taken from Cyganowski's $\mathrm{PhD}$ thesis, are displayed with the distributions of the $\mathrm{CH}_{3} \mathrm{OH} 44 \mathrm{GHz}$ emission (purple, + symbols), $24 \mu \mathrm{m}$ thermal dust emission (yellow contours), and the $\mathrm{CH}_{3} \mathrm{OH} 6.7 \mathrm{GHz}$ emission (black diamonds) superimposed on a GLIMPSE color image. Here, we see that the $6.7 \mathrm{GHz}$ emission is located precisely toward the central source (presumably the YSO responsible for driving the associated outflow), the $44 \mathrm{GHz}$ emission is distributed along the extended green $4.5 \mu \mathrm{m}$ emission, and

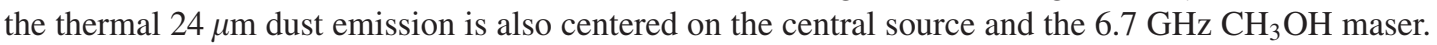
This is consistent with what one should expect from the different excitation mechanisms. The $24 \mu \mathrm{m}$ emission and $6.7 \mathrm{GHz}$ masers are located near the central source where the radiation field is the most intense and the $44 \mathrm{GHz}$ maser is located along the presumed outflows where they are expected to collide with the ambient ISM. Finally, in Figure 2 (center and right) [2], CO outflows are shown as red and blue contours superimposed on GLIMPSE color images that trace the $4.5 \mu \mathrm{m}$ excess emission of two EGOs rather closely. The sum of all these data plus some HCO+ profiles (not shown due to space constraints) strongly supports the interpretation that EGOs are in fact massive protostars in the act of rapid accretion.

Since the GLIMPSE surveys will cover the entire Galactic plane $\left(360^{\circ}\right)$ out to $\pm 1^{0}$ in latitude (more at some longitudes), it is possible to identify a substantial fraction of all massive YSOs within a degree of the Galactic plane. So we now know where to concentrate our efforts to learn more about the details of the earliest stages of massive star formation.

\section{INFRARED DARK CLOUDS (IRDCS)}

Another major feature of the disk of our Galaxy at infrared wavelengths is the prevalence of IRDCs. This is not a surprise because IRDCs are nothing more than the densest parts of molecular clouds that were imaged at radio wavelengths years ago. They are opaque at $8 \mu \mathrm{m}$ indicating that $\mathrm{A}_{V}>70$ mag. Figure 3 (left) shows an example of an IRDC seen in silhouette against the Galactic background emission at $8 \mu \mathrm{m}$. Several studies of IRDCs have been undertaken in molecular lines at radio wavelengths, references to which are given by [5]. Typical properties of IRDCs are as follows: they are cold, T $\sim 20 \mathrm{~K}$; they are dense, $\mathrm{n}\left(\mathrm{H}_{2}\right) \sim 10^{5} \mathrm{~cm}^{-3}$; and they are the locations where stars are forming (see [5] and references therein). Ammonia emission and $8 \mu \mathrm{m}$ extinction are essentially coincident, thus permitting a good determination of the gas temperature, density, and velocity structure in IRDCs. The close association of $\mathrm{NH}_{3}$ emission and $8 \mu \mathrm{m}$ extinction is illustrated in Figure 3 (right).

\section{DIFFUSE PAH EMISSION}

When examining large panoramic segments of the GLIMPSE Galactic plane, one is struck by the wide distribution of diffuse $8 \mu \mathrm{m}$ emission, which is largely dominated by PAHs. PAHs, of course, are very bright in the PDR shells that surround HII regions (massive star formation regions). However, there is also very clearly diffuse PAH emission well beyond the PDRs of HII regions. As noted above, PAHs are effectively destroyed by hard UV radiation within HII regions, so the excitation of PAHs in the diffuse ISM is controlled by soft UV that can pass through the H-ionization fronts of HII regions, which is probably mostly supplied by B and possibly A stars that are far more numerous than O stars. In Figure 4, 

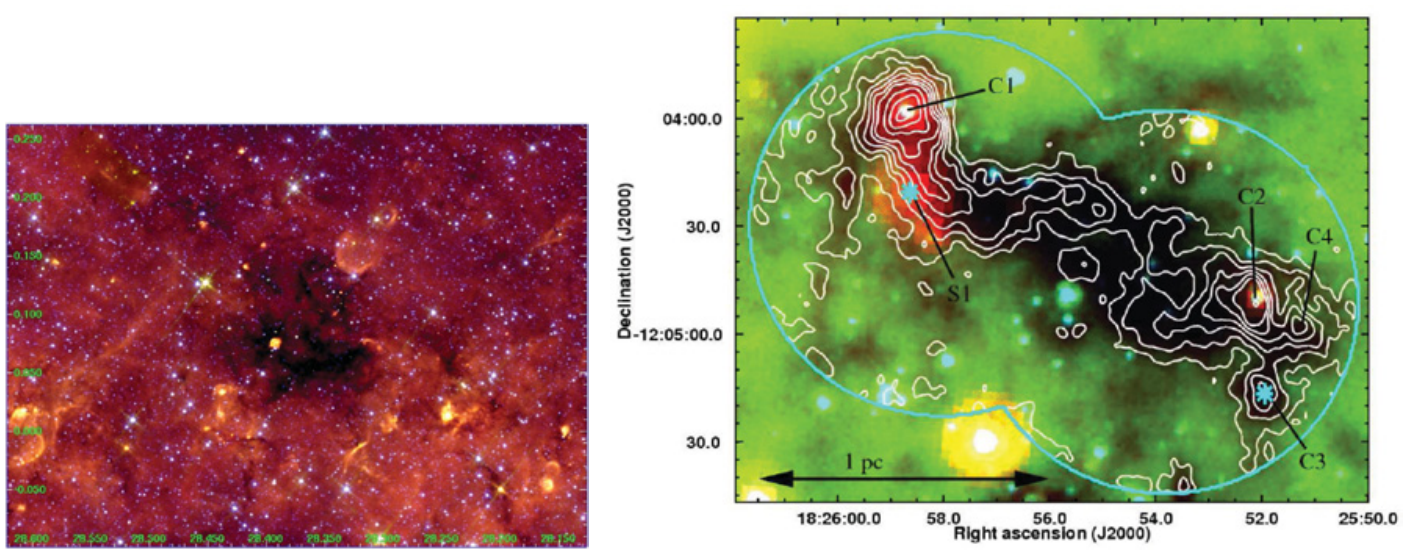

Figure 3. (left): A GLIMPSE 3 color image (red- $8 \mu \mathrm{m}$ : green-5.8 $\mu \mathrm{m}$ : blue-3.6 $\mu \mathrm{m}$ ) showing an IRDC silhouetted against the diffuse background emission. Note the bright YSO near the left boundary of the IRDC. $($ right $)$ : $\mathrm{NH}_{3}(1,1$; main hf line) contours from the VLA (resolution 4.4"x3.4") superimposed on a GLIMPSE./MIPSGAL image (red$24 \mu \mathrm{m}$ : green- $8 \mu \mathrm{m}$; blue- $4.5 \mu \mathrm{m}$ ) of the IRDC G1930+0.07 (from Devine et al. 2011). C1-4 indicate $\mathrm{NH}_{3}$ peaks and asterisks indicate $\mathrm{H}_{2} 2 \mathrm{O}$ masers. Note the tight correspondence of $\mathrm{NH}_{3}$ emission and $8 \mu \mathrm{m}$ extinction and the correspondence between MIR peaks and/or $\mathrm{H} 2 \mathrm{O}$ masers with $\mathrm{NH}_{3}$ peaks.

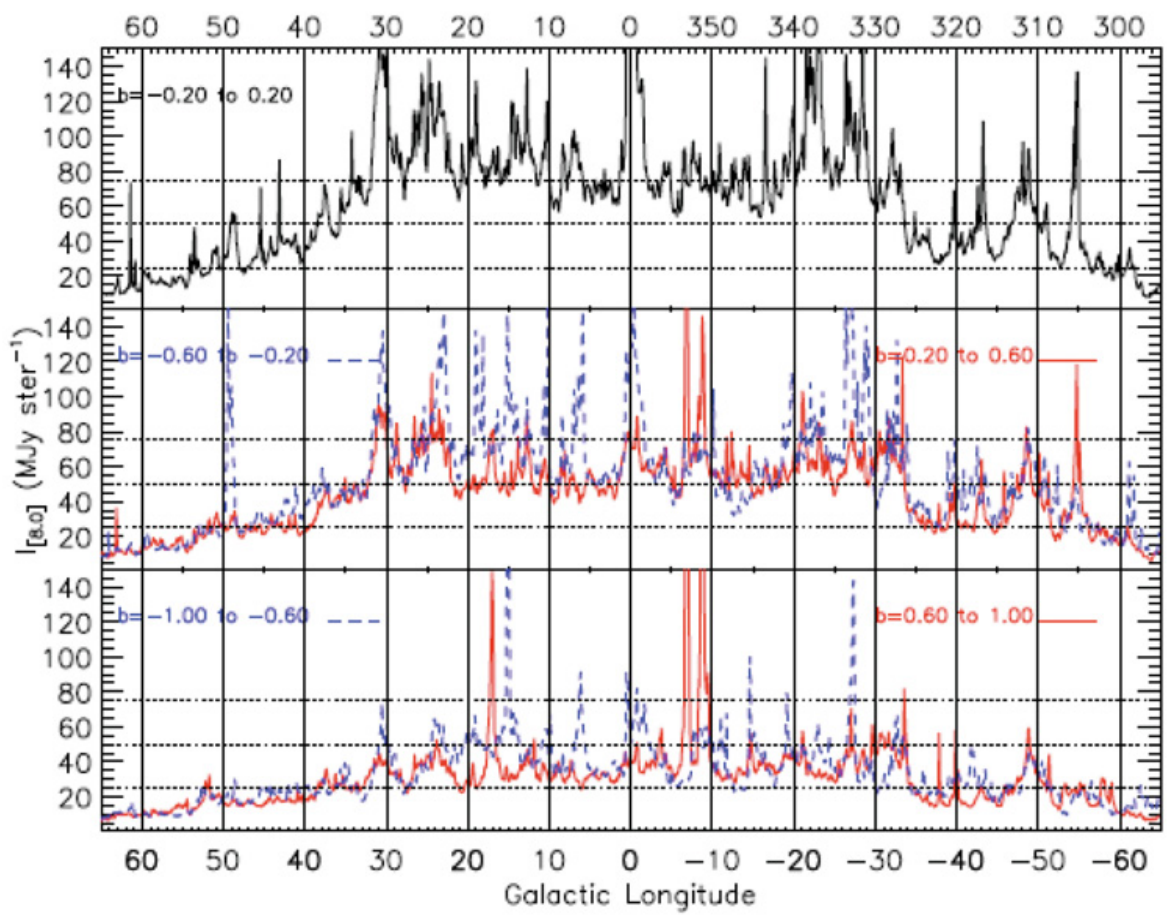

Figure 4. $8 \mu \mathrm{m}$ emission profiles averaged over $0.4^{0}$ wide slices in Galactic latitude from $+65^{0}$ to $-65^{0}$ in longitude. The top panel is centered on $0^{0}$ latitude. The middle panel are slices centered at $-0.4^{0}$ latitude (blue) and $+0.4^{0}$ latitude (red). The bottom panel are slices centered at $-0.8^{0}$ latitude (blue) and $+0.8^{0}$ latitude. Note the flatness of the profiles in the approximate interval $\pm 30^{\circ}$ longitude. The spikes are not noise but are places where HII region PDRs occur. The basic shapes of the profiles are very similar above and below the plane at equal distances from the plane. 
the brightness of the $8 \mu \mathrm{m}$ band is shown as a function of Galactic longitude averaged over $0.4^{0}$ wide slices in latitude. The black (top panel) is centered on $b=0^{0}$, in the middle and bottom panels blue represents $0.4^{0}$ wide averages below the plane $\left(-0.2^{0}\right.$ to $0.6^{0}$ and $-0.6^{0}$ to $\left.-1.0^{0}\right)$ and red represents $0.4^{0}$ wide averages over the same latitude segments to the north of the plane. The spikes in these plots are real; they occur where the strip passes through an HII region. The signal/noise in these plots is greater than 10 everywhere. These profiles do not look like the distribution of the old red giant stellar population, which exponentially increases toward the Galactic center and is even steeper inside $\pm 10^{\circ}$ due to the nuclear bulge.

The approximate constant brightness inside $\pm 30^{0}$ longitude is a surprise and is obviously telling us about the soft UV radiation field in the inner Galaxy. The fall off beyond $30^{\circ}$ from the Galactic center is not so clear. Is it because PAH abundances decline beyond $30^{\circ}$ or is the radiation field too soft to excite diffuse PAH emission? This is something we may be able to get a handle on by applying stellar models of the Galaxy to see what is required to fit the observed PAH profiles. If the predicted stellar radiation field becomes too soft to excite PAHs, then it could well be that PAHs are widely distributed in the Galaxy, even where they are not seen in emission, and may well represent a substantial reservoir of complex hydrocarbon molecules in the Galactic plane.

\section{SUMMARY}

I have given an abbreviated summary of a few selected topics that have come to light from the GLIMPSE/MIPSGAL Spitzer surveys of the Galactic plane. These are topics that are of particular interest to me. Another reviewer could easily have chosen a different set of topics (objects) to discuss, as these surveys are rich in data that have not received the attention required to obtain a deeper understanding of them and their relationship to the structure and physics of the Milky Way.

I have shown that the disk of the Milky Way is rife with MIR bubbles and that dust is present and relatively well mixed with the ionized hydrogen. The presence of the dust, even in high temperature, low density, wind-shocked hydrogen requires a continuous source of dust. It is suggested that the sources of the dust may be from slowly photoevaporating cold, dense, pre-existing cloudlets of ISM which have been over-run by the expanding hydrogen ionization front. Photoevaporating disks around lower mass stars, similar to the proplids near the Trapezium, may also contribute dust to the nebula. Whatever the origin, it was shown that the presence of dust in hot, shocked $\mathrm{H}+$ gas is the dominant coolant and significantly reduces the gas temperature below levels that would otherwise make these regions strong $\mathrm{X}$-ray sources. Also the total internal energy is significantly reduced below that of dust- free nebulae, resulting in significantly altered evolution of wind-blown HII regions.

Rapidly accreting massive protostars show up in the GLIMPSE color images as slightly extended green objects when the $4.5 \mu \mathrm{m}$ IRAC band is tagged as green. The $\mathrm{CH}_{3} \mathrm{OH} 6.7 \mathrm{GHz}$ transition is located on the central protostar where the MIR radiation field is intense and the $\mathrm{CH}_{3} \mathrm{OH} 44 \mathrm{GHz}$ transition is distributed along the extended $4.5 \mu \mathrm{m}$ emission which traces the shocked bipolar outflows where they crash into the ambient ISM. CO outflows are also coincident with the $4.5 \mu \mathrm{m}$ emission. All the evidence so far supports the hypothesis that EGOs are protostars in the act of rapidly accreting mass to become massive stars, but in a phase prior to becoming a HC or UC HII region, as they have no detectable radio continuum emisson. The GLIMPSE survey provides a powerful way to identify all massive protostars within $8-10 \mathrm{kpc}$ of the Sun when the $4.5 \mu \mathrm{m}$ band is tagged as green.

IRDCs are easily detected at $8 \mu \mathrm{m}$ silhouetted against the background $8 \mu \mathrm{m}$ emission. These regions are cold, dense, and located in the central regions of $\mathrm{CO}$ molecular clouds. Their opaqueness at $8 \mu \mathrm{m}$ implies visual extinctions $\mathrm{A}_{V} \geq 70$ mags. MIR images show that IRDCs typically have only a small fraction of their volume undergoing star formation at any given time, which raises the question of what supports them. The large number of IRDCs also raises questions of their formation and lifetimes. 
The GLIMPSE survey has shown that diffuse $8 \mu \mathrm{m}$ emission is widely distributed in the Galactic plane. Since the $8 \mu \mathrm{m}$ IRAC band is typically dominated by PAH emission, it is suggested that PAHs are widely distributed in the disk of the Galaxy which poses unanswered questions of their origin, abundance, chemistry, and excitation.

The work reported here was made possible with input from John Everett (bubbles), Claudia Cyganowski (EGOs), and the GLIMPSE core team (Brian Babler, Marilyn Meade, Bob Benjamin, and Barb Whitney).

\section{References}

[1] Churchwell, E. et al., ApJ 649, 2006, 759

[2] Cyganowski, C., PhD Thesis, University of Wisconsin 2010

[3] Davis, C.J., MNRAS, 374, 2007, 29

[4] De Buizer, J. M. \& Vacca, W. D., AJ 140, 2010, 196

[5] Devine, K. E. et al. ApJ, 733, 2011, 44

[6] Everett, J. E. \& Churchwell, E. ApJ 713, 2010, 592

[7] Ferland, G. J. et al. PASP 110, 1998, 761

[8] Morisset, C., IAU Symp. 234, Planetary Nebulae in Our Galaxy and Beyond, eds. M. J. Barlow \& R. H. Mendez, Cambridge Univ. Press, Cambridge, 2006, 467

[9] Smith, M. D. \& Rosen, A. MNRAS 357, 2005, 1370

[10] Ybarra, J. E. \& Lada, E. A. ApJ 695, 2009, L120

[11] Ybarra, J. E. et al. ApJ 714, 2010, 469 